\title{
FERIAS DE AGRO-BIODIVERSIDAD CUBANAS: VÍA PARA LA SELECCIÓN DE VARIEDADES DE TOMATE ${ }^{1}$
}

\author{
Carlos Celedonio Moya-López ${ }^{2}$,Erik Orozco-Crespo², María Elena Mesa-Fleitas ${ }^{3}$
}

\begin{abstract}
RESUMEN
Ferias de agro-biodiversidad cubanas: vía para la selección de variedades de tomate. El objetivo de este trabajo fue determinar en ferias de agro-biodiversidad el aporte de los agricultores en la selección de variedades de tomate (Solanum lycopersicon L.) mejor adaptadas a las características de una localidad o sistema productivo específico. La investigación se efectuó en el Instituto Nacional de Ciencias Agrícolas, San José de Las Lajas, Cuba, en la campaña 2008-2009, siguiendo los métodos tradicionales para la evaluación de variedades; posteriormente, en una feria de agro biodiversidad en el municipio Batabanó, campaña 20102011, se tuvieron en cuenta los criterios de los productores sobre las variedades estudiadas. La evaluación estadística se centró en el análisis descriptivo de las variables, análisis de varianza, formación de conglomerados y uso de tablas de contingencia. Los resultados del ensayo comparativo se cotejaron con los de la feria de agro-biodiversidad. Los productores para discriminar las variedades, no solo tuvieron en cuenta las variables relacionadas con el rendimiento, sino que consideraron otras relacionadas con su experiencia en el cultivo y la preferencia personal.
\end{abstract}

Palabras clave: selección participativa, fitomejoramiento participativo, elección de especies.

\begin{abstract}
Cuban agro-biodiversity fairs: a way to select tomato varieties. The aim of this study was to determine, in agrobiodiversity fairs, farmers' contribution regarding the selection of tomato varieties (Solanum lycopersicon L.) better adapted to the characteristics of a locality or specific productive system. The research was carried out at the National Institute of Agricultural Sciences, San José de Las Lajas, Cuba, in 2008-2009 season, applying the traditional methods to assess varieties; subsequently, at an agro-biodiversity fair in Batabanó, in 2010-2011 season, taking into account the producers criteria on the varieties studied. The statistical evaluation was focused on the descriptive analysis of the variables, analysis of variance, conglomerate formation, and use of contingency tables. The results of the comparative test were crosschecked to the data from the agro-biodiversity fair. In order to discriminate varieties, producers took into account not only the variables related to the performance, but also others related to their farming expertise and personal preferences.
\end{abstract}

Keywords: participatory selection, participatory plant breeding, species choice.

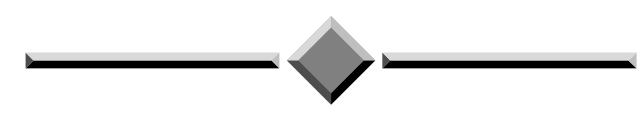

1 Recibido: 17 de marzo, 2015. Aceptado: 8 de octubre, 2015. Este trabajo formó parte del proyecto de investigación titulado "Sistemas locales de producción de semillas: estudio de casos municipios San José de las Lajas, Rodas y Gibara”, financiado por el Ministerio de Ciencia, Tecnología y Medio Ambiente de Cuba (CITMA).

2 Universidad Técnica del Norte, Facultad de Ingeniería en Ciencias Agropecuarias y Ambientales y Facultad de Ingeniería en Ciencias Aplicadas. Avenida 17 de julio \# 5-21 y General José María Córdova, Ibarra, Imbabura, Ecuador. carlosmoya47@gmail.com (autor para correspondencia),erikcopo@gmail.com

3 Universidad de las Américas, Facultad de Formación General, Avenida Los Granados y Colime, Quito, Pichincha. Doctora en Ciencias de la Información.maria.mesa@udlanet.ec 


\section{INTRODUCCIÓN}

El tomate (Solanum lycopersicon L.) se encuentra entre los cultivos hortícolas de mayor importancia comercial en el mundo, debido a su alta demanda y a la gran utilización en la dieta de la población, tanto para consumo fresco como en conservas (Prohens y Nuez, 2008). No obstante, su bajo rendimiento en la mayoría de los países tropicales se debe al efecto negativo que ejercen los factores climáticos, fundamentalmente las altas temperaturas, lluvias y humedad relativa elevada; además la incidencia de plagas y enfermedades (FAOSTAT, 2009). También debe agregarse el empleo por los productores de semillas de baja calidad, variedades poco adaptadas a las condiciones de la localidad y uso de tecnologías de cultivo no apropiadas a las características agroecológicas del sitio de siembra aspectos que encuentran solución mediante la capacitación que reciben y ponen en práctica en el proceso de ejecución de las ferias de agro-biodiversidad (Dueñas et al., 2007).

El comportamiento diferenciado de las variedades en los distintos ambientes se conoce como interacción genotipo ambiente (GxA), que se acentúa cuando los ambientes de selección y destino son diferentes, entre las que sobresalen las relaciones suelo-planta en condiciones tropicales (Martínez-Viera y Dibut, 2009).

La modalidad participativa en el fitomejoramiento que incluye la participación de los productores y otros actores locales, en todo el proceso productivo, la cual tiene como actividad final la ejecución de la feria de agro-biodiversidad, ofrece una solución a este problema, pues permite seleccionar las variedades más adaptadas al ambiente de destino y definir las tecnologías más adecuadas a la localidad, las cuales permitan la obtención de productos agrícolas de alta demanda que satisfagan la preferencia de los usuarios (Fé de la et al., 2010). El productor decide si una variedad específica resulta útil para las formas habituales de cultivo; esta decisión requiere la comprensión integral de las necesidades humanas que se intentan satisfacer con su introducción en un sistema productivo específico (Lores et al., 2008).

El empleo de los métodos de fitomejoramiento participativo, específicamente las ferias de agrobiodiversidad para la selección de variedades de tomate, ha tenido éxito en todo el territorio cubano, apoyado fundamentalmente por la experiencia y el conocimiento adquirido por los productores en los trabajos de selección de variedades, producción de semillas y manejo agronómico del cultivo (Álvarez et al., 2008; Moya et al., 2009).

Los métodos participativos de mejoramiento genético en el cultivo del tomate, están siendo aprovechados por instituciones de investigación y docencia, lo que ha permitido generalizar su empleo en los trabajos de mejoramiento genético, extensionismo agrario y la enseñanza de pre y posgrado en tomate y otros cultivos como el frijol (Phaseolus vulgaris L.) y la papa (Solanum tuberosum L.) (Fé de la et al., 2009; Fé de la et al., 2007; Lambert et al., 2012).

Los resultados se logran por la acción combinada de los fitomejoradores, productores y otros actores locales, que facilitan un mayor flujo de diversidad a las comunidades, se facilita la capacitación de los diferentes actores en los procesos productivos, tecnologías de producción, selección de variedades y producción y conservación de las semillas (Ortiz et al., 2008)

Dada la necesidad de continuar incrementando la diversidad genética del tomate en manos de los productores, y la imposibilidad de que los centros de investigación científica, puedan cubrir todas las necesidades de las comunidades productivas, se requirió desarrollar investigaciones en localidades del occidente de Cuba. El objetivo de este trabajo fue determinar en ferias de agro-biodiversidad el aporte de los agricultores en la selección de variedades de tomate (Solanum lycopersicon L.) mejor adaptadas a las características de una localidad o sistema productivo específico.

\section{MATERIALES Y MÉTODOS}

El ensayo comparativo de variedades en la provincia de Mayabeque, correspondiente a la región occidental, se realizó en el Instituto Nacional de Ciencias Agrícolas (INCA), situado en los $22^{\circ} 24^{\prime}$ de latitud norte y $79^{\circ} 58^{\prime}$ de longitud oeste, a 138 msnm, en la localidad de San José de las Lajas, Cuba (Academia de Ciencias de Cuba, 1989). Este ensayo se llevó a cabo en la campaña 2008-2009.

Los tipos de suelos predominantes en esta región son el ferralítico rojo compactado (Hernández et al., 1999). Las semillas se trataron con imidacloprid (1-(6-cloro-3-piridilmetil)-N-nitroimidazolidin-2- 
ylideneamina) a razón de $80 \mathrm{~g}$ del producto por $\mathrm{kg}$ de semilla, disuelto en $200 \mathrm{ml}$ de agua, con el objetivo de evitar el ataque temprano del virus del encrespamiento amarillo de la hoja del tomate (TYLCV). Las demás labores culturales se ejecutaron según las instrucciones técnicas para el cultivo.

En el ensayo comparativo se incluyeron tres variedades comerciales y nueve líneas procedentes del programa de mejoramiento genético del INCA (Cuadro 1). Se evaluó el rendimiento por área (t/ha), rendimiento por planta $(\mathrm{kg} / \mathrm{planta})$, peso por fruto $(\mathrm{g})$ y número de frutos por planta. El diseño experimental de campo fue de bloques completos al azar con cuatro repeticiones y el área de las parcelas fue de $15,4 \mathrm{~m}^{2}$, formadas por dos surcos de veinte plantas cada uno; el experimento se plantó en el mes de noviembre del 2008.

Cuadro 1. Variedades de tomate incluidas en el ensayo comparativo de rendimiento. Instituto Nacional de Ciencias Agrícolas (INCA), provincia Mayabeque, Cuba. Campaña de siembra 2008-2009.

Table 1. Tomato varieties included in the comparative performance test. National Institute of Agricultural Sciences (INCA), Mayabeque, Cuba, 2008-2009 planting season.

\begin{tabular}{lll}
\hline No. & Variedad & Origen \\
\hline 1 & Mariela & Variedad comercial \\
2 & Amalia & Variedad comercial \\
3 & Línea 1 & INIFAT-28xNC-NBR-2 \\
4 & Línea 41 & PeraxNC-NBR-2 \\
5 & Línea 35 & PeraxNC-NBR-2 \\
6 & Mara & Variedad comercial \\
7 & Línea-44 & PeraxNC-NBR-2 \\
8 & Línea-24 & PeraxNo. 24x A-1-1 \\
9 & Línea-14 & INIFAT-28xNC-NBR-2 \\
10 & Línea-38 & PeraxNC-NBR-2 \\
11 & Línea-42 & PeraxNC-NBR-2 \\
12 & Línea-43 & PeraxNC-NBR-2 \\
\hline
\end{tabular}

Del ensayo comparativo se seleccionaron la totalidad de las variedades para ser incluidas en la feria de agro-biodiversidad en la localidad de Batabanó, en la misma provincia de Mayabeque, situado a $\operatorname{los} 22^{\circ} 15^{\prime}$ de latitud norte y $82^{\circ} 30^{\prime}$ de longitud oeste y una altura de 100 msnm (Academia de Ciencias de Cuba, 1989). Esta se realizó en la campaña 2010-2011.
En la feria de agro-biodiversidad se utilizó como diseño el denominado de "parcelas simples", de 182 $\mathrm{m}^{2}$ por cada una de las doce variedades, las mismas estuvieron compuestas por catorce surcos de $10 \mathrm{~m}$ de largo, separados a 1,40 m entre ellos, para un total de 433 plantas por parcela.

Como resultado de la convocatoria a participar en la feria, participaron en la misma un total de 31 actores locales, de los cuales la mayoría eran productores directos, además de tres especialistas del Ministerio de Agricultura (Minagri) y dos dirigentes de la Asociación Nacional de Agricultores Pequeños (ANAP).

Además de las características incluidas en el ensayo comparativo de rendimiento, los participantes en la feria agregaron la respuesta de las plantas al ataque de plagas y enfermedades, el color del fruto maduro y la compactación del mismo.

Para el análisis de la información obtenida en la feria, se utilizó el listado de los participantes, una planilla donde ellos escribieron sus nombres, el lugar de procedencia y anotaban los números de las cinco variedades que seleccionaron; además, señalaron con una cruz las características que utilizaron como criterio de selección. Con esa información se definieron las variedades más seleccionadas, se tomó en cuenta la influencia del género y la función social de los participantes (Fé de la et al., 2010).

Para el análisis estadístico de los resultados del ensayo comparativo de variedades, se estudiaron las variables siguientes:

- Variedad: variable nominal que identificó el nombre de la variedad bajo estudio.

- Rendimiento por hectárea: variable de escala medida en toneladas por hectárea.

- Rendimiento por planta: variable de escala medida en kilogramos por planta.

- Peso del fruto: variable de escala medida en gramos.

- Frecuencia de frutos por planta: variable de escala que caracterizó la representatividad de cada variedad en cuanto al número total de frutos por planta.

Para el análisis descriptivo de las variables de escala se emplearon la media y la desviación típica como medidas de tendencia central y de dispersión, respectivamente. Para la descripción del comportamiento de las mismas en nivel bajo, medio y alto, se emplearon los percentiles $33,33 \%$ y 
$66,66 \%$, los cuales permitieron dividir el rango en tres categorías (Sáez, 2010).

Se realizó un análisis de varianza de clasificación doble para los caracteres de rendimiento y número de frutos por planta, mientras que para el peso promedio de los frutos se empleó un diseño completamente aleatorizado con un tamaño de muestra de diez frutos por variedad.

Se formaron conglomerados que permitieran agrupar las variedades estudiadas en grupos internamente homogéneos. Todo ello utilizando el comportamiento promedio de las variedades en cada una de las variables de escala anteriormente mencionadas.

Para determinar el número de conglomerados se empleó, primeramente, un procedimiento jerárquico con el objetivo de explorar la cantidad de grupos a formar. Posteriormente, se aplicó un procedimiento no jerárquico (conglomerado de k-medias) para determinar finalmente los conglomerados de pertenencia de cada una de las variedades analizadas.

Los análisis relacionados con la formación de conglomerados jerárquicos se basaron fundamentalmente en la tabla de historial de conglomeración y en el dendograma. Se aplicó el "Método del codazo" para detectar el mayor salto en los coeficientes de conglomeración, de manera tal que permitiera explorar la cantidad de conglomerados a formar (Thorndike, 1953); método también empleado por Lee et al. (2013). Se transformaron las variables en puntuaciones $\mathrm{Z}$, en vez de trabajar con los datos originales.

Una vez formados y descritos los conglomerados había que determinar si existía algún tipo de preferencia o nivel de aceptación por parte de los productores sobre algún conglomerado en particular, es decir, sobre las variedades que componen dichos conglomerados. Se probó el nivel de preferencia que tuvieron los productores sobre las variedades que actualmente se están comercializando. Para el procesamiento de esta información se empleó el análisis de tablas de contingencia bidimesionales, para el cual se emplearon las siguientes variables:

Conglomerado de pertenencia: variable nominal que identifica el conglomerado de pertenencia de cada una de las variedades estudiadas.

Comercialización: variable nominal que identifica si la variedad se está comercializando o no en la actualidad.

Nivel de aceptación: variable de escala que describe el nivel de aceptación de los productores sobre las variedades estudiadas, medida sobre la base porcentual de la cantidad de productores que votan a favor de una determinada variedad.

Para el cruce de variables de escala con variables nominales se empleó el coeficiente $\mathrm{Eta}^{4}$, la prueba exacta de Monte Carlo y un nivel de significación del 5\%.

\section{RESULTADOS Y DISCUSIÓN}

\section{Análisis de varianza}

Los intervalos de comportamiento obtenidos del análisis descriptivo se muestran en el Cuadro 2.

Los resultados de los análisis de varianza realizados en el ensayo comparativo de rendimiento (Cuadro 3) mostraron que las variedades más productivas fueron Mariela y Amalia, con rendimientos de 47,3 y 45,0 t/ ha, respectivamente, sin diferencias significativas con la variedad Mara y las líneas 1, 41, 35, 44, 24, 14 y 38. La línea 42 no mostró diferencias significativas con las variedades Mariela y Amalia, cuando se analizó el rendimiento por planta, incluyéndose en el grupo que mostró frutos con mayor peso promedio, no se debe descartar la línea 43, la cual solo presentó diferencias significativas con la variedad Mariela en el carácter rendimiento por planta y el peso promedio de sus frutos fue superior a $100 \mathrm{~g}$.

El comportamiento de Amalia, Mariela y Mara concuerda con los resultados de otros autores que las han evaluado en similares condiciones (Álvarez et al., 2007). En relación con el peso promedio de los frutos, siete de las variedades superaron los $100 \mathrm{~g}$ como promedio, sobresaliendo la línea 14 con 127 g, la cual no mostró diferencias estadísticas con las líneas 1, 38, 42 y 44; esta característica hace que esas líneas sean, en principio, muy aceptables para productores y consumidores.

Los resultados de las ferias de agro-biodiversidad indicaron que en Mayabeque (Cuadro 4) los participantes mostraron preferencia por las líneas y variedades expuestas, en el siguiente orden: línea 44, línea 43, línea 35, que fueron seleccionadas por 24,23 y 20 votos respectivamente, de 31 posibles.

Estos resultados coinciden con los del ensayo comparativo de rendimiento, en lo que respecta a

\footnotetext{
4 Cuyo cuadrado puede interpretarse como la proporción de la varianza de la variable de escala que puede ser explicada a partir de la variable categórica empleada.
} 
Cuadro 2. Intervalos de confianza para el comportamiento de las variables de escala. Universidad Técnica del Norte, Ibarra, Ecuador. Noviembre-diciembre 2014

Table 2. Confidence intervals to measure the performance of scale variables. Technical University of the North, Ibarra, Ecuador. November-december 2014.

\begin{tabular}{lccc}
\hline Variables & \multicolumn{3}{c}{ Intervalos de comportamiento } \\
\cline { 2 - 4 } & Bajo & Medio & Alto \\
\hline Rendimiento (t/ha) & {$[0 ; 36,27]$} & $(36,27 ; 42,57]$ & $(42,57 ; \infty)$ \\
Rendimiento (kg/planta) & {$[0 ; 1,29]$} & $(1,29 ; 1,48]$ & $(1,48 ; \infty)$ \\
Peso del fruto (g) & {$[0 ; 95,00]$} & $(95,00 ; 111,33]$ & $(111,33 ; \infty)$ \\
Frecuencia frutos por planta & {$[0 ; 0,0701]$} & $(0,0701 ; 0,0902]$ & $(0,0902 ; \infty)$ \\
Nivel de aceptación & {$[0 ; 0,15]$} & $(0,15 ; 0,35]$ & $(0,35 ; \infty)$ \\
\hline
\end{tabular}

Cuadro 3. Resultados obtenidos en el análisis de varianza (ANOVA) del ensayo comparativo de rendimiento. Instituto Nacional de Ciencias Agrícolas (INCA), San José de las Lajas, provincia Mayabeque, Cuba. Junio, 2009.

Table 3. Results from the Analysis of Variance (ANOVA) from the comparative test of performance. National Institute of Agricultural Sciences (INCA), San José de las Lajas, Mayabeque, Cuba. June, 2009.

\begin{tabular}{cccccc}
\hline No. & $\begin{array}{c}\text { Variedad/ } \\
\text { Línea }\end{array}$ & $\begin{array}{c}\text { Rendimiento } \\
(\mathbf{t} / \mathbf{h a})\end{array}$ & $\begin{array}{c}\text { Rendimiento } \\
\text { (kg/planta) }\end{array}$ & $\begin{array}{c}\text { Peso } \\
\text { fruto }(\mathbf{g})\end{array}$ & $\begin{array}{c}\text { Frutos/ } \\
\text { planta }\end{array}$ \\
\hline 1 & Mariela & $47,3 \mathrm{a}$ & $1,66 \mathrm{a}$ & $82 \mathrm{e}$ & $15 \mathrm{a}$ \\
2 & Amalia & $45,0 \mathrm{a}$ & $1,58 \mathrm{ab}$ & $82 \mathrm{e}$ & $13 \mathrm{abc}$ \\
3 & Línea 1 & $42,8 \mathrm{ab}$ & $1,50 \mathrm{ab}$ & $112 \mathrm{abc}$ & $11 \mathrm{abc}$ \\
4 & Línea 41 & $42,7 \mathrm{ab}$ & $1,49 \mathrm{ab}$ & $106 \mathrm{bcd}$ & $12 \mathrm{abc}$ \\
5 & Línea 35 & $42,3 \mathrm{ab}$ & $1,34 \mathrm{ab}$ & $97 \mathrm{cde}$ & $12 \mathrm{abc}$ \\
6 & Mara & $41,5 \mathrm{abc}$ & $1,45 \mathrm{ab}$ & $94 \mathrm{de}$ & $12 \mathrm{abc}$ \\
7 & Línea-44 & $38,8 \mathrm{abc}$ & $1,20 \mathrm{ab}$ & $119 \mathrm{ab}$ & $8 \mathrm{c}$ \\
8 & Línea-24 & $37,2 \mathrm{abc}$ & $1,37 \mathrm{ab}$ & $80 \mathrm{e}$ & $14 \mathrm{ab}$ \\
9 & Línea-14 & $35,8 \mathrm{abc}$ & $1,26 \mathrm{ab}$ & $127 \mathrm{a}$ & $9 \mathrm{bc}$ \\
10 & Línea-38 & $35,3 \mathrm{abc}$ & $1,40 \mathrm{ab}$ & $110 \mathrm{abc}$ & $10 \mathrm{bc}$ \\
11 & Línea-42 & $31,5 \mathrm{bc}$ & $1,10 \mathrm{ab}$ & $113 \mathrm{abc}$ & $8 \mathrm{c}$ \\
12 & Línea-43 & $29,2 \mathrm{c}$ & $1,02 \mathrm{~b}$ & $102 \mathrm{bcd}$ & $9 \mathrm{bc}$ \\
& $\overline{\mathrm{x}}$ & 39,1 & 1,36 & 102 & 11 \\
& EE $(\overline{\mathbf{x}})$ & 1,04 & 0,04 & 2,39 & 0,41 \\
& Significación & $\mathrm{p}<0,001$ & $\mathrm{p}<0,01$ & $\mathrm{p}<0,0001$ & $\mathrm{p}<0,0001$ \\
\hline
\end{tabular}

Medias con letras iguales no difieren significativamente para Duncan $\mathrm{p}<0,05 /$ Means followed by the same letters do not differ significantly by Duncan $p<0,05$. 
Cuadro 4. Resultados de la selección participativa de variedades de tomate en la feria de agrobiodiversidad. Municipio Batabanó, provincia Mayabeque, Cuba. Campaña de siembra 20102011.

Table 4. Results of participatory selection of tomato varieties in the agro-biodiversity. fair. Batabanó, Mayabeque, Cuba. 2010-2011 planting season.

\begin{tabular}{lccc}
\hline No. & $\begin{array}{c}\text { Variedad o } \\
\text { línea }\end{array}$ & $\begin{array}{c}\text { Total de } \\
\text { votos }\end{array}$ & $\begin{array}{c}\text { \% de participantes } \\
\text { que votan por la } \\
\text { línea }\end{array}$ \\
\hline 1 & Línea-44 & 24 & 77 \\
2 & Línea-43 & 23 & 74 \\
3 & Línea 35 & 20 & 65 \\
4 & Línea 1 & 11 & 35 \\
5 & Mara & 11 & 35 \\
6 & Línea-42 & 9 & 29 \\
7 & Línea-41 & 8 & 26 \\
8 & Línea 38 & 6 & 19 \\
9 & Amalia & 4 & 13 \\
10 & Mariela & 4 & 13 \\
11 & Línea 24 & 3 & 10 \\
12 & Línea 14 & 1 & 3 \\
\hline
\end{tabular}

las líneas 44, 35, 1 y la variedad Mara, las cuales se incluyeron en el grupo de mayores rendimientos y, con excepción a la línea 35, fueron del grupo de mayor peso promedio de los frutos; de ellas la número 1 también estuvo entre las de mayor número de frutos por planta.
Los resultados de la línea 43, indicaron que esta no mostró altos rendimientos por área en el ensayo comparativo, y fue la segunda más seleccionada en la feria, dado que tuvo frutos con peso promedio superiores a los $100 \mathrm{~g}$, lo que la hizo más atractiva para los productores de la localidad y suministradores de tomate a los mercados de la capital (Álvarez et al., 2008). Además, sus rendimientos por planta relativamente altos le facilitaron una mejor adaptación a las condiciones del suelo y clima de la localidad de Batabanó y fue superior a los rendimientos por planta alcanzados en la localidad de San José de las Lajas (Lores et al., 2008).

\section{Formación de conglomerados y análisis de las tablas de contingencia}

La cantidad de conglomerados sugeridos por la aplicación del método jerárquico se muestra en el Cuadro 5. Como puede observarse se combinaron varios métodos de conglomeración con varias medidas de distancia, tanto de similaridad como de disimilaridad.

Se deduce que las medidas de similaridad fueron más estables en la cantidad de conglomerados sugeridos, mientras que las medidas de disimilaridad fueron más inestables sugiriendo entre tres y cinco conglomerados. Es válido resaltar que la formación de cinco conglomerados quedó descartada, debido a la poca cantidad de variedades analizadas en el estudio.Los resultados anteriores se tomaron como insumo principal para la formación del conglomerado de k-medias.

Cuadro 5. Cantidad de conglomerados sugeridos por la combinación de métodos de conglomeración y medidas de distancia. Universidad Técnica del Norte, Ibarra, Ecuador. Noviembre-diciembre. 2014.

Table 5. Amount of conglomerates suggested by the combination of cluster methods and distance measurements. Technical University of the North, Ibarra, Ecuador. November-December, 2014.

\begin{tabular}{|c|c|c|c|c|c|}
\hline Método de conglomeración & $\begin{array}{l}\text { Vinculación } \\
\text { inter-grupos }\end{array}$ & $\begin{array}{l}\text { Vinculación } \\
\text { intra-grupos }\end{array}$ & $\begin{array}{c}\text { Vecino más } \\
\text { próximo }\end{array}$ & $\begin{array}{c}\text { Vecino más } \\
\text { lejano }\end{array}$ & $\begin{array}{c}\text { Método de } \\
\text { Ward }\end{array}$ \\
\hline & \multicolumn{5}{|c|}{ Medidas de similaridad } \\
\hline Correlación de Pearson & 3 & 3 & 3 & 3 & - \\
\hline \multirow[t]{2}{*}{ Coseno } & 3 & 3 & 3 & 3 & \\
\hline & \multicolumn{5}{|c|}{ Medidas de disimilaridad } \\
\hline Euclídea al cuadrado & 5 & 3 & 6 & 5 & 3 \\
\hline Minkowsky & 5 & 5 & 6 & 5 & 3 \\
\hline Chebychev & 5 & 4 & 9 & 3 & 3 \\
\hline
\end{tabular}


Cuadro 6. Centro de los conglomerados finales. Universidad Técnica del Norte, Ibarra, Ecuador. Noviembrediciembre, 2014.

Table 6. Center of final conglomerates. Technical University of the North, Ibarra, Ecuador, November-December, 2014.

\begin{tabular}{lccc}
\hline Variables & \multicolumn{3}{c}{ Conglomerado } \\
\cline { 2 - 4 } & $\mathbf{1}$ & $\mathbf{2}$ & $\mathbf{3}$ \\
\hline Rendimiento (t/ha) & 42,75 & 36,20 & 39,13 \\
Rendimiento (kg/planta) & 1,52 & 1,27 & 1,32 \\
Peso del fruto (g) & 84,50 & 105,60 & 119,33 \\
Frecuencia frutos/planta & 0,1015 & 0,0767 & 0,0702 \\
\hline
\end{tabular}

Los elementos fundamentales sobre los cuales se basaron los análisis fueron: el historial de las iteraciones, pertenencia a los conglomerados, los centros de los conglomerados finales, las distancias entre dichos centros y el número de variedades por conglomerado.

Los resultados del procesamiento de los datos para la formación de tres conglomerados se muestran en los Cuadros 6 y 7.

Se destacan como otros resultados importantes, deducidos del conglomerado de k-medias, que la convergencia total se alcanzó favorablemente en la segunda iteración; las variedades más alejadas del centro de sus conglomerados de pertenencia fueron la línea 35 del conglomerado 2 y la variedad Mara del conglomerado 1; los conglomerados más diferentes entre sí fueron el 1 y el 3, con una medida de distancia de 35,021; esto a pesar de que los conglomerados resultaron estar compuestos por cantidades semejantes de variedades.

Al describir los conglomerados formados, y de acuerdo a los intervalos de confianza obtenidos (Cuadro 2), puede decirse que:

El conglomerado 1: se caracterizó por altos rendimientos, tanto en toneladas por hectárea como en kilogramos por planta, bajo peso del fruto y alto número de frutos por planta.

El conglomerado 2: presentó bajos rendimientos, tanto en toneladas por hectárea como en kilogramos por planta, y un comportamiento medio en cuanto a peso del fruto y número de frutos por planta.

El conglomerado 3: se caracterizó por valores medios en cuanto al rendimiento, frutos de gran peso y bajo número de frutos por planta.

Las variedades que componen el conglomerado 1 se sugieren para áreas de producción con condiciones
Cuadro 7. Pertenencia de las variedades de tomate a cada uno de los conglomerados. Universidad Técnica del Norte, Ibarra, Ecuador. Noviembre-Diciembre 2014.

Table 7. Tomato varieties belonging to each one of the conglomerates. Technical University of the North, Ibarra, Ecuador. November-December, 2014.

\begin{tabular}{lccc}
\hline $\begin{array}{l}\text { Número de } \\
\text { caso }\end{array}$ & Variedad & Conglomerado & Distancia \\
\hline 1 & Mariela & 1 & 5,194 \\
2 & Amalia & 1 & 3,364 \\
3 & Línea 1 & 3 & 8,201 \\
4 & Línea 41 & 2 & 6,516 \\
5 & Línea 35 & 2 & 10,544 \\
6 & Mara & 1 & 9,582 \\
7 & Línea-44 & 3 &, 487 \\
8 & Línea-24 & 1 & 7,147 \\
9 & Línea-14 & 3 & 8,360 \\
10 & Línea-38 & 2 & 4,493 \\
11 & Línea-42 & 2 & 8,768 \\
12 & Línea-43 & 2 & 7,875 \\
\hline
\end{tabular}

de cultivo menos idóneas, donde los productores demuestren mayor interés por los rendimientos y buen comportamiento a condiciones adversas para el cultivo. A este conglomerado pertenecen todas las variedades comerciales que fueron incluidas en el experimento y la línea 24 , la cual es no comercial y que indica la posibilidad de incluirla en futuros trabajos de extensión agraria.

Las variedades que componen el conglomerado 3 son preferibles para zonas que suministran productos para mercados más exigentes en cuanto a los parámetros de calidad del fruto, y donde los precios están directamente relacionados con los mismos. Las variedades que componen el conglomerado 2 poseen un bajo y medio perfil, por lo que no se recomiendan para su uso comercial, pero si para futuras evaluaciones en otras condiciones de cultivo.

Para detectar posibles relaciones entre las variables constituidas por los conglomerados formados y el nivel de aceptación de los productores en las ferias de agrobiodiversidad, fue empleada la tabla de contingencia que se muestra en el Cuadro 8, y cuyos resultados se concretan en el Cuadro 9.

Las variedades que conformaron el conglomerado 2 fueron las que mayor nivel de aceptación tuvieron 
Cuadro 8. Tabla de contingencia para relacionar las variables nivel de aceptación con conglomerado de pertenencia. Universidad Técnica del Norte, Ibarra, Ecuador. Noviembre-diciembre, 2014.

Table 8. Contingency table to relate variables of level of acceptance and conglomerate belonging. Technical University of the North, Ibarra, Ecuador. November-December, 2014.

\begin{tabular}{|c|c|c|c|c|c|c|c|c|c|c|c|c|c|c|}
\hline & & \multicolumn{10}{|c|}{ Nivel de aceptación } & \multirow{2}{*}{ Total } & \multirow{2}{*}{$\bar{x}$} & \multirow{2}{*}{$\mathbf{S}$} \\
\hline & & 0,03 & 0,10 & 0,13 & 0,19 & 0,26 & 0,29 & 0,35 & 0,66 & 0,74 & 0,77 & & & \\
\hline \multirow{3}{*}{$\begin{array}{l}\text { Conglomerado de } \\
\text { pertenencia }\end{array}$} & 1 & 0 & 1 & 2 & 0 & 0 & 0 & 1 & 0 & 0 & 0 & 4 & 0,1775 & 0,0579 \\
\hline & 2 & 0 & 0 & 0 & 1 & 1 & 1 & 0 & 1 & 1 & 0 & 5 & 0,4260 & 0,1119 \\
\hline & 3 & 1 & 0 & 0 & 0 & 0 & 0 & 1 & 0 & 0 & 1 & 3 & 0,3833 & 0,2143 \\
\hline
\end{tabular}

Cuadro 9. Medida direccional para caracterizar la dependencia entre las variables, nivel de aceptación y conglomerado de pertenencia. Universidad Técnica del Norte, Ibarra, Ecuador. Noviembre-diciembre, 2014

Table 9. Directional measurement to characterize the dependence between the variables of level of acceptance and conglomerate belonging. Technical University of the North, Ibarra, Ecuador. November-December, 2014.

\begin{tabular}{|c|c|c|c|}
\hline & & & Valor \\
\hline \multirow{2}{*}{$\begin{array}{l}\text { Nominal } \\
\text { por intervalo }\end{array}$} & \multirow{2}{*}{ Eta } & Conglomerado de pertenencia (3) dependiente & 0,843 \\
\hline & & Nivel de aceptación dependiente & 0,455 \\
\hline
\end{tabular}

$(42,60 \%)$ (Cuadro 8). En él, la variedad de menor aceptación fue la línea 38 (19\%). Le siguió el conglomerado 3 con un nivel alto de aceptación, de un $38,33 \%$. Es válido destacar que la desviación típica en este conglomerado demostró la diversidad de criterios de los productores en cuanto a la aceptación de las variedades, teniéndose a la línea 44 con el más alto nivel de aceptación (77\%) y a la línea 14 con el más bajo (3\%). El conglomerado 1 fue el de más bajo nivel de aceptación $(17,75 \%)$, teniéndose a la variedad Mara con un nivel más alejado del resto de las variedades que lo conforman (35\%).

Los niveles de aceptación no quedaron totalmente explicados por los conglomerados de pertenencia, lo que se explica en el moderado valor alcanzado en el coeficiente Eta $(0,455)$ (Cuadro 9), que indicó que los conglomerados solo explicaron el $21 \%$ de la varianza observada en el nivel de aceptación.

Los productores a la hora de realizar la votación, no solo consideraron las variables de rendimiento contempladas en el presente estudio, sino que incluyeron también otras relacionadas con las características cualitativas de la planta y del fruto, su experiencia en el cultivo de estas variedades y su preferencia personal.

Para caracterizar la relación existente entre las variables nivel de aceptación de los productores en las ferias de agro-biodiversidad y la comercialización actual de la variedad, fue empleada la tabla de contingencia que se muestra en el Cuadro 10, acompañada de sus respectivos resultados en el Cuadro 11.

Las variedades no comerciales recibieron un nivel de aceptación superior a las variedades que actualmente se están comercializando, con un 37,56\% (nivel alto) y un $20,33 \%$ (nivel medio) de los votos totales, respectivamente (Cuadro 10). En la contingencia se observó que la variable nivel de aceptación no quedó bien explicada por la variable comercialización. Esta débil relación se justifica por el bajo valor del coeficiente Eta $(0,306)$ (Cuadro 11) y que además indicó que la comercialización solo explicó el 9\% de la varianza observada en el nivel de aceptación.

Los productores del municipio no seleccionaron como favoritas a las variedades que actualmente se están comercializando, prefiriendo aquellas variedades 
Cuadro 10. Tabla de contingencia para relacionar las variables nivel de aceptación con comercialización. Universidad Técnica del Norte, Ibarra, Ecuador. Noviembre-Diciembre, 2014.

Table 10. Contingency table to relate variables of level of acceptance and commercialization. Technical University of the North, Ibarra, Ecuador. November-December, 2014.

\begin{tabular}{|c|c|c|c|c|c|c|c|c|c|c|c|c|c|c|}
\hline & & \multicolumn{10}{|c|}{ Nivel de aceptación } & \multirow{2}{*}{ Total } & \multirow{2}{*}{$\bar{x}$} & \multirow{2}{*}{$\mathbf{S}$} \\
\hline & & 0,03 & 0,10 & 0,13 & 0,19 & 0,26 & 0,29 & 0,35 & 0,66 & 0,74 & 0,77 & & & \\
\hline \multirow{2}{*}{ Comercialización } & $\begin{array}{l}\text { No comercial } \\
\end{array}$ & 1 & 1 & 0 & 1 & 1 & 1 & 1 & 1 & 1 & 1 & 9 & 0,3756 & 0,0924 \\
\hline & Comercial & 0 & 0 & 2 & 0 & 0 & 0 & 1 & 0 & 0 & 0 & 3 & 0,2033 & 0,0733 \\
\hline
\end{tabular}

Cuadro 11. Medida direccional para caracterizar la dependencia entre las variables nivel de aceptación y comercialización. Universidad Técnica del Norte, Ibarra, Ecuador. Noviembre-Diciembre, 2014.

Table 11. Directional measurement to characterize the dependence between the variables of level of acceptance and commercialization. Technical University of the North, Ibarra, Ecuador. November-December, 2014.

\begin{tabular}{|c|c|c|c|}
\hline & & & Valor \\
\hline \multirow{2}{*}{$\begin{array}{l}\text { Nominal por } \\
\text { intervalo }\end{array}$} & \multirow{2}{*}{ Eta } & Comercialización dependiente & 0,882 \\
\hline & & Nivel de aceptación dependiente & 0,306 \\
\hline
\end{tabular}

no comerciales y que proceden del programa de mejoramiento genético del INCA. A lo que se añade que no todos los participantes en la feria habían estado estrechamente relacionados con las variedades incluidas en el experimento, y donde el nivel educacional y el origen laboral de los mismos pueden estar marcando dichas preferencias.

\section{LITERATURA CITADA}

Academia de Ciencias de Cuba. 1989. Nuevo atlas nacional de Cuba. Instituto Cubano de Geodesia y Cartografía. La Habana-Madrid, CUB.

Álvarez, M., C. Moya, D. Plana, F. Dueñas, M. Varela, F. Llerena, y H. Ríos. 2007. Incremento de la diversidad de variedades de tomate, su adopción y diseminación por los productores en la comunidad El Tejar-La Jocuma, La Palma, Pinar del Río. Cultivos Trop. 28(2):71-77.

Álvarez, M., C. Moya, J. Rodríguez, y D. Plana. 2008. Claudia, Mercy y Mayle, tres nuevas variedades de tomate para el consumo fresco. Cultivos Trop. 29(1):43-43.
Dueñas, F., C. Moya, M. Álvarez, D. Plana, H. Ríos, I. Amat, y L. Arias. 2007. Incremento de la diversidad de variedades de tomate, su adopción y diseminación por los productores en la Comunidad El Tejar La Jocuma, La Palma, Pinar del Río. Cultivo Trop. 28(2):71-77.

Fé de la, C., J.G. Castillo, J.L. Salomón, A. Caballero, y N. Lorenzo. 2007. La selección participativa de variedades (SPV) en el cultivo de la papa. Cultivos Trop. 28(3):77-82.

Fé de la, C., C. Moya, J. Arzuaga, y E. Fonseca. 2010. Coincidencia en la selección participativa de variedades de tomate y la selección por rendimiento en una feria de agrobiodiversidad. Cultivos Trop. 31(2):92-96.

Fé de la, C., O. Rodríguez, M. Ponce, y R. Ortíz. 2009. Coincidencia en la selección participativa de variedades de frijol común y la selección por rendimiento en una feria de agro-biodiversidad. Cultivos Trop. 30(2):3-79.

FAOSTAT. 2009. Food and agricultural commodities production: top production - Cuba - 2007. http://faostat. fao.org/site/339/default.aspx (accessed 5 jan. 2010).

Hernández, A., J.M. Pérez, D. Bosch, L. Rivero, E. Camacho, J. Ruíz, y J.M. Ruíz de León. 1999. Nueva versión de clasificación genética de los suelos de Cuba. Ministerio de la Agricultura, La Habana, CUB.

Lambert, T., L. Rodríguez, M. Rivero, y B. Castro. 2012. El fitomejoramiento participativo como herramienta para la selección de variedades de tomate (Solanum lycopersicon L.). Rev. Cienc. Agric. 29(2):53-66.

Lee, P.H., Y.Y. Yu, I. McDowell, G.M. Leung, and T.H. Lam. 2013. A cluster analysis of patterns of objectively measured physical activity in Hong Kong. Public Health Nutr. (Popular Republic China) 16:1436-1444.

Lores, A., A. Leyva, y T. Tejeda. 2008. Evaluación espacial y temporal de la agrobiodiversidad en los sistemas campesinos de la comunidad "Zaragoza" en La Habana. Cultivos Trop. 29(1):3-10.

Martínez-Viera, R., y B. Dibut. 2009. Utilización de nuevos paradigmas que permitan profundizar los 
conocimientos sobre las relaciones suelo-planta en condiciones tropicales. Cultivos Trop. 30(2):5-9.

Moya, C., M. Álvarez, D. Plana, M. Florida, F. Dueñas, J. Arzuaga, J. Hernández, L. Amat, J. Sanstisteban, y E. Fonseca. 2009. Evaluación y selección participativa de nuevas líneas y variedades de tomate (Solanum lycopersicon) en la región Oriental de Cuba. Cultivos Trop. 30(2):66-72.

Ortiz, R., H. Ríos, M. Ponce, C. Gill, P. Chaveco, y O. Rodríguez. 2008. Impacto del fitomejoramiento participativo en el contexto de la innovación local evaluada por los propios actores involucrados. En: I Simposio de desarrollo endógeno, innovación, innovación local y extensionismo agrario. INCA, 2428 nov. 2008. CUBA.

Prohens, J., and F. Nuez. 2008. Handbook of plant breeding. Vegetables II: Fabaceae, Liliaceae, Solanaceae, and Umbelliferae. Springer Science, USA.

Sáez, J.A. 2010. Métodos estadísticos con R y R commander. Universidad de Jaén, ESP.

Thorndike, R.L. 1953. Who belongs in the family?. Psychometrika (Popular Republic China) 18:267-276. 